Original Article

\title{
Detailed Checklist of the Grammitid Specimens from the Biological Museum Herbarium under VNU University of Science (HNU)
}

\author{
Phan Ke Loc ${ }^{1, *}$, Nguyen Cong Toan ${ }^{2}$, Le Hong Thom ${ }^{3}$ \\ ${ }^{1}$ VNU University of Science, 334 Nguyen Trai, Hanoi, Vietnam \\ ${ }^{2}$ Faculty of Biology Biological Museum, VNU University of Science, \\ 17 Le Thanh Tong, Hoan Kiem, Hanoi, Vietnam \\ ${ }^{3}$ VNU University of Education, 144 Xuan Thuy, Cau Giay, Hanoi, Vietnam
}

Received 04 January 2020

Revised 13 April 2020; Accepted 13 April 2020

\begin{abstract}
This study aims to compile a detailed checklist of grammitid specimens kept in the Biological Museum Herbarium under VNU University of Science (HNU). The study adopted the Pteridophyte Phylogeny Group classification (PPG I 2016) to classify these specimens and fifteen references were used to identify their scientific names. Detailed field labels in HNU provided various and rich data for compiling the checklist. The study analyzed 43 collection field numbers and identified 16 species of 7 genera. The richest in species was Oreogrammitis with 5 species; two genera (Prosaptia and Scleroglossum), each with 3 species; one genus (Calymnodon) with two species; and three other genera (Cteropterella, Themelium and Tomophyllum), each with one species.
\end{abstract}

Keywords: Detailed checklist, field labels, grammitids, PPG I (2016), HNU.

\section{Introduction}

Grammitidoideae Parris \& Sundue is a subfam. of the family Polypodiaceae J.Presl \& C.Presl with 33 genera and an estimated 911 species [1]. They are ferns usually small, often pendulous, epiphytes and lithophytes, occasionally terrestrial; rhizomes erect and radial with stipes in whorls, or \pm creeping and dorsiventral with stipes in two rows; stipes glabrous or with hairs of various kinds, rarely with scales at the base; laminae simple to tripinnatifid, glabrous or with hairs of various types; sori without indusial; sporangia glabrous

\footnotetext{
* Corresponding author.

Email address: pkeloc@yahoo.com

https://doi.org/10.25073/2588-1140/vnunst.4987
} 
or with simple non-septate hairs. Grammitidoideae have worldwide distribution with more than 20 genera and over 750 species, in the wet tropics of both hemispheres. They usually are epiphytic, sometimes lithophytic, less commonly terrestrial, mostly growing with bryophytes, Hymenophyllaceae family and other small epiphytic ferns [2].

\section{Materials and Methods}

Materials: Making detailed checklist of all 43 grammitid collecting numbers kept in HNU, the herbarium of the Biological Museum of the VNU University of Science (HUS) is our goal. Traditional morphological methods are used to examine and description of external morphology, photographic illustration, collecting all other data related to each research sample.

Carefully field lables enable us rich and various data to make detailed checklist. Then we compare the characteristics of the specimens in 2 research genera with valid names (Protologue), and related documents to identify its proper name. Among 43 collecting numbers of 16 species in 7 genera are listed. They are arranged in alphabetically order of groups, then of genera.

Methods: The geographical distribution of studied specimens is arranged in floristic provinces, from $\mathrm{N}$ to $\mathrm{S}$ : NEFP- acronym of the North Eastern Floristic Province, NIFPacronym of the North Indochinese Floristic Province, CTFP-acronym of the Central Truong Son Floristic Province, STFP- acronym of the South Truong Son Floristic Province and SIFPacronym of the South Indochinese Floristic Province [3]. Fifteen main references are used to identify scientific names of studied specimens [4-14].

\section{Results}

\subsection{Pragmatic key to genera}

1a. Fronds more than $9-10 \mathrm{~cm}$ tall .2 (Tall group)

1b. Fronds less than $8-9 \mathrm{~cm}$ tall ...3 (Dwarf group) 2a. Stipe has hairs, articulated to phyllopodia ........................ 1. Prosaptia

2b. Stipe glabrous, not articulated to phyllopodia ........................ 2.Themelium

3a. Fronds simple ...................... 4

3 b. Fronds pinnate or pinnately divided ...5

4a. Sori linear, \pm paralellel to midvein and sunken in groove ............. 3. Scleroglossum

4b. Sori \pm circular to broadly elliptic, superficial or slightly sunken in broad shallow depressions in lamina.......... 4. Oreogrammitis

5a. Sori protected by folded pinna, one per pinna................................ 5. Calymnodon

$5 b$. Sori not protected by folded pinna, one or more per pinna ............................ 6

$6 a$. Two rows of sori on longest pinnae or, if only one row then rhizome scales absent ......6. Tomophyllum

6b. One sorus per pinna, or if two, in one row .......................... 7. Ctenopterella

3.2. Key to species of all genus

3.2.1. Key to species of the genus Calymnodon

1a. Lamina glabrous or sparcely hairy; hairs ca. 0.1-0.2 mm long; sterile pinnae at base, oblong, ca. 2-3 x $0.9 \mathrm{~mm}$........ 1. C. asiaticus

1b. Lamina with frequent hairs on both surfaces; hairs ca. $1 \mathrm{~mm}$ long; sterile pinnae at base, narrowly obovate, ca. 3 x $1.5 \mathrm{~mm}$ .....................................2. C. gracilis

3.2.2. Key to species of the genus Oreogrammitis

1a. Laminae glabrous........... 3. O. nuda

1b. Laminae hairy....................... 2

2a. Lateral veins distinct ... 4. O. reinwardtii

2b. Lateral veins hidden ................. 3

3a. Laminae usually more than $6 \mathrm{~cm}$ long; stipe usually more than $1 \mathrm{~cm}$ long; stipe hairs up to $1.2 \mathrm{~mm}$ long; lateral veins 1 or 2forked........................... 1. O. congener

3b. Laminae usually less than $6 \mathrm{~cm}$ long; stipe usually less than $1 \mathrm{~cm}$ long....................... 4

4a. Stipe hairs dark reddish brown, 0.2-1.8 $\mathrm{mm}$ long....................... 5. O. sinohirtella

4b. Stipe hairs dark brown, 0.1-0.2 mm long..............................2. O. dorsipila

3.2.3. Key to species of the genus Prosaptia 
1a. Sori oblique or parallel to plane of lamina 3. P. contigua

1b. Sori perpendicular to plane of lamina .2

2a. Rim of sori higher on side nearest costa 1. P. intermedia

2b. Rim of sori even height ...2. P. obliquata

3.2.4. Key to species of the genus Scleroglossum

1a. Soral grooves opening oblique to surface of laminae

2a. Laminae not clearly narrowed below sori 1. S. pusillum

2b. Laminae clearly narrowed below sori 2. S. puxidatum

1b. Soral grooves opening perpendicular to surface of laminae 3. S. sulcatum

\subsection{Detailed checklist of studied species}

It is arranged in Tall group and in Dwarf group; genera in each group in alphabetically order. Data of each species are presented in following order: Nomenclature section, morphological description, habitat and ecology, geographical distribution are separated by sign:.-

\subsubsection{Genus Prosaptia}

3.3.1.1. Prosaptia contigua (G.Forst.) C.Presl, Tent. Pterid. 166. 1836. Basionym: Trichomanes contiguum G.Forst., Fl. Ins. Austr. 84. 1786. Type: French Polynesia, Oceania, Tahiti, Society Islands - Windward, 11 June 1996, David Nelson s.n. (Lectotype BM 000036058!). Synonym: Ctenopteris contigua (G.Forst.) Holttum, Rev. Fl. Malaya 2: 230, fig. 130. 1954. Type: C. Seram, Manusela National Park, along a trail between Wae (River) Ansela and Wae Huhu, Kecamatan (District) Seram Utara, Indonesia, 03 Jan 1985, M.Kato, B.Sunarno and H.Akiyama (Isotype $\mathbf{L}$ 0700179!).- Stipes short, 1-2.5 cm long with occasional to scattered hairs, less than $0.7 \mathrm{~mm}$ long; lamina linear-elliptic, $14-20 \times 1.8-2.7 \mathrm{~cm}$; lamina pinnate or deeply pinnately divided to narrow wing less than $0.8 \mathrm{~mm}$ wide along rachis, $38-52$ (or more) pairs, 0.8-1.5 × 0.1-0.2 cm; margins of pinnae entire when sterile, lobed to wing less than $1 \mathrm{~mm}$ wide along pinna midvein when fertile; sorus 1 per tooth and/or 1 at apex of pinna, 1-4 on each pinna, sunken in marginal urceolate cavities opening outward, parallel to plane of lamina, lamina margin forming of pit mouth, rim of cavity slightly with some short simple hairs on both sides. In primary mossy wet coniferous forest with Fokienia hodginsii along main ridge. Occasional an epiphytic on mossy trees.- STFP. Lam Dong Prov., Lac Duong Distr., Da Chais Comm., around point $12^{\circ} 05^{\prime} 27^{\prime}$ ' $\mathrm{N}, 108^{\circ} 39^{\prime} 43^{\prime \prime} \mathrm{E}$, at elevation 2100-2300 m, along main ridge of Bi Doup mountain. Aver. et al. HLF 5267b. 15 Oct 2005.

3.3.1.2. Prosaptia intermedia (Ching) Tagawa, J. Jap. Bot. 25(6): 115. 1950. Basionym: Polypodium urceolare var. intermedium Ching, Bull. Dept. Biol. Sun Yatsen Univ. 6: 32. 1933. Typus: Yaoshan, Kwangsi, Tuesday, February 28, 1928, Sin (Holotype PE 01863726!, 01863727!). Synonym: Prosaptia urceolaris var. intermedia (Ching) Ching, Bull. Fan Mem. Inst. Biol., Bot. 10(1): 17. 1940.- Stipe short, less than $1.5 \mathrm{~cm}$, with hairs dense, $0.5-1 \mathrm{~mm}$; lamina narrowly elliptic in outline, $15-30 \times 1.9-2.5 \mathrm{~cm}$; pinnae ascending, narrowly triangular oblong to narrowly oblong, 6-16 × 2-4 mm; sori circular to broadly elliptic, deeply sunken in cavities in lamina with rim less than $0,6 \mathrm{~mm}$, higher on side nearest costa, slightly prominent on adaxial surface, less than 7 in a medial row on each side of costa.- NEFP. Ha Giang Prov., Dong Van Distr, Ta Lung Comm, vicinities of Ha De Vill., $23^{\circ} 12^{\prime} 23^{\prime \prime} \mathrm{N}, 105^{\circ} 17^{\prime} 18^{\prime \prime} \mathrm{E}$, at elevation $1450 \mathrm{~m}$, degraded primary coniferous forest with Pseudotsuga sinensis on very steep rocky slopes and along tops of narrow rocky remnant Chong Sia ridge composed with highly eroded marblelike limestone. Epiphytic fern on very steep rocky shady mossy slope. Common. Aver. et al. HAL 8619. 15 Dec 2005; Yen Minh Distr, Lao Va Chai Comm., vicinities of Chi Sang Vill., Ha Giang Prov., Yen Minh Distr, Lao Va Chai Comm., vicinities of Chi Sang Vill., around point $23^{\circ} 06^{\prime} 20^{\prime}$ ' $\mathrm{N}, 105^{\circ} 04^{\prime} 25^{\prime}$ ' $\mathrm{E}$, at elevation about $1450 \mathrm{~m}$. Primary mossy coniferous forest with Tsuga sinensis on tops of high rocky ridge composed with highly eroded marble-like 
limestone. Epiphyte on mossy trees along rocky mossy ridge edge. Common. Aver. et al. HAL 8416. 9 Dec 2005; Vi Xuyen Distr., Phong Quang Comm., Man Vill., limestone peaks and ridges NE of Vill., 22 53 '21" N 104 $56^{\prime} 45^{\prime \prime} \mathrm{E}$, at elevation 595-787 m. Rare, lithophytic, in shade. Sporangia brown. Harder et al. DKH et al. 6359. 15 Feb 2001 \& Harder et al. DKH 6379 et al., 15 Feb 2001; Bac Me Distr., Phieng Luong Comm., around Phieng Luong Vill., about $4 \mathrm{~km}$ to SW of Phieng Luong Comm., 22³9'29" N, 105¹9'35" E. Slightly logged primary closed evergreen broad-leaved forest on slopes of remnant mountains composed with solid highly eroded crystalline and partially shale limestone at elevation 1150-1250 m. Epiphytic in shady place. Not rare. Aver. et al. HAL 6422. 04 Mar 2005; Tuyen Quang Prov., Na Hang Distr., Sinh Long Comm., Khuoi Phin Vill., 22038'19.8" N, $105^{\circ} 20^{\prime} 26.9^{\prime \prime} \mathrm{E}$, at elevation 1100-1200 m. In closed primary evergreen broad-leaved mixed with conifers, lowland forests on ridge and top of limestone mountain. Epiphyte herb, $50 \mathrm{~cm}$ tall. Common. P.V.The et al. CPC 4532. 28 Sep 2011 \& NS Khang CPC 4595. 29 Sep 2011; Ham Yen, Yen Thuan, Cao Duong Vill., 22 ${ }^{\circ} 16 ' 51.6^{\prime \prime}$ $\mathrm{N}, 105^{\circ} 00^{\prime} 13.7^{\prime \prime} \mathrm{E}$, at elevation $910 \mathrm{~m}$. In slightly affected primary limestone foest. Epiphyte, clinging on big and moist tree trunk; common. PVThe \& TN Bon TB 114, 21 Apr 2019.- NIFP. Dien Bien Prov., Tua Chua Distr., Sin Chai Comm., 22 ${ }^{\circ} 03^{\prime} 38^{\prime \prime} \mathrm{N} 103^{\circ} 19^{\prime} 56^{\prime \prime}$ E. In primary humid evergreen broad-leaved forest on very steep rocky slopes and on tops of remnant mountain composed with highly eroded marblelike solid crystalline limestone at elevation 1350-1500 m. Clustering epiphyte on old mossy trees in shady places. Common. Aver. et al. CPC 932. 14 Dec 2010.- Hoa Binh Prov., Mai Chau Distr., Pa Co Comm., near Small Pa Co Vill., Hoa Binh Prov., Mai Chau Distr., Pa Co Comm., near Small Pa Co Vill., 204' N, 104 ${ }^{0} 54^{\prime \prime}$ E, at elevation 1100-1200 m, on limestone mountain. Epiphytic in heavily logged primary closed evergreen coniferous with the dominance of Fokienia hodginsii submontane forests on ridges of mountains. PK Loc et al. P6983 \& P6999A. 04 Jun 1995.- CTFP. Thua Thien-Hue Prov., A
Luoi Distr., Hong Van Comm., Ta Lo Vill., $16^{\circ} 21^{\prime} 52^{\prime \prime} \mathrm{N}, 107^{\circ} 09^{\prime} 36^{\prime \prime} \mathrm{E}$, at elevation about $1000-1100 \mathrm{~m}$. Secondary wet closed broadleaved forest on very steep mountain slopes composed with shale and granite along shady stream canyon. Lithophytic and epiphytic on mossy trees along stream shady rocky slope. Locally common. Aver. et al. HAL 7667. 30 Apr 2005; Huong Thuy Distr., Duong Hoa Comm., Huong Thuy forest enterprise territory, Huong Thuy Distr., Duong Hoa Comm., Huong Thuy forest enterprise territory, around point $16^{\circ} 13^{\prime} 23^{\prime \prime} \mathrm{N}, 107^{\circ} 35^{\prime} 16^{\prime \prime} \mathrm{E}$, SW slopes of Mang Chan ridge at elevation about $350-500 \mathrm{~m}$. Fractionally logged primary closed evergreen broad-leaved forest along steep stream slopes composed with shale with quartzite. Epiphytic and lithophytic fern on wet shady rocks along rocky stream canyon. Locally common. Aver. et al. HAL 8310. 11 May 2005.- Kontum Prov., Kon Plong Distr., Hieu Comm., Mang La forest enterprise, $14^{\circ} 39^{\prime} \mathrm{N}, 108^{\circ} 25^{\prime}$ E. Primary wet mossy mountain coniferous forest on sandstone (with Pinus dalatensis, Fokienia hodginsii, Dacrydium elatum, Dacrycarpus imbricatus) along ridge tops at elevation $1200 \mathrm{~m}$. Epiphyte on mossy trees in shady wet places. Lithophytic and epiphytic on mossy trees along stream shady rocky slope and rocky mossy ridge edge. Common. Aver. et al. VH 5302. 18 Apr 2000.

3.3.1.3. Prosaptia obliquata (Blume) Mett., Reise Novara 1: 214. 1870. Basionym: Polypodium obliquatum Blume, Enum. Pl. Javae 2: 128. 1828. Synonym: Ctenopteris obliquata (Blume) Copel., Philip. J. Sci. sl 111, 1953. Typus: Asia-Tropical, Indonesia, Java, Blume s.n. (Syntype L 0700187!, 0700188!).- Stipe long, 2-4.5 cm, with hairs frequent to dense, less than $0.5 \mathrm{~mm}$; lamina narrowly elliptic in outline, $15.5-32 \times 1.5-4 \mathrm{~cm}$; middle pinnae largest, linear to oblong-lanceolate, $0.8-2.2 \times 0.15-0.35 \mathrm{~cm}$; sori sunken perpendicular to plane of lamina in steep sided pits, eliptic to narrowly eltiptic, oblique cavities, rim of cavity distinctly and evenly prominent, not fringed with hairs; row medial on each side of costa, less than 7 pairs on 1 pinna. Primary broad-leaved evergreen closed wet mossy cloudy forest on crystalline highly 
metamorphosed sandstone. Epiphytic on mossy trees and lithophytic on mossy boulders. Common.- SIFP. Dak Nong Prov., Dak Gloong Distr., Dak P'Lao Comm., steep moutain slopes along main ridge of Ta Xung mountain system at elevation 1700-1985 m, around point of highest peak at $11^{\circ} 51^{\prime} 30^{\prime \prime} \mathrm{N} 108^{\circ} 02^{\prime} 08^{\prime \prime}$ E. Aver. et al. HLF 5670. 08 Nov 2005.

\subsubsection{Genus Themelium}

3.3.2.1. Themelium halconense (Copel.) Parris, Kew Bull. Vol. 59(2), 225, 2004. Basionym: Polypodium halconense Copel., Philipp. J. Sci., C. Bot. 2: 138 (1907). Type: Philippines, Mindoro, Mt Halcon, elev. 2300 m, November 1906, Merrill 5976 (lectotype MICH 1115951!). Synonym: Ctenopteris halconensis (Copel.) Copel., Fern Fl. Philipp. 3: 533, 1960.Stipe short, less than $3 \mathrm{~cm}$, hairless; lamina narrowly elliptic in outline, $5.5-15.5 \times 1-4 \mathrm{~cm}$; pinnae at apex entire to rarely crenulate and slightly pinnately $1 / 4-1 / 2$ from magins to veins, middle pinnae pinnately $1 / 3-2 / 3$ from magins to veins, linear to narrowly oblong, $0.5-2.5 \times 0.05$ $0.3 \mathrm{~cm}$, pinnae at base abruptly shortened to some obliquely orbicular or deltoid; pinnules triangular, indistinct, extending beyond sorus or not, $0.2-1.2 \times 0.3-0.5 \mathrm{~mm}$, with 1 vein per fertile pinnule, usually with 1 or 2 hairs on apical margin, sometime on middle margin; hais simple, dark brown, absent from both surfaces of lamina; sori orbicular, superficial, 1 at base of pinnule, not mouth protruding. In primary mossy wet coniferous forest with Fokienia hodginsii along main ridge of mountains. Lithophytic and epiphytic on mossy trees.- STFP. Lam Dong Prov., Lac Duong Distr., Da Chais Comm., around point $12^{\circ} 05^{\prime} 27^{\prime \prime} \mathrm{N} 108^{\circ} 39^{\prime} 43^{\prime \prime} \mathrm{E}$, at elevation 2100-2300 $\mathrm{m}$, along main ridge of $\mathrm{Bi}$ Doup mountain. Primary mossy wet coniferous forest with Fokienia hodginsii along main ridge of mountains. Lithophytic and epiphytic on mossy trees. Locally common. Aver. et al. HLF 5256. 15 Oct 2005 [15].

\subsubsection{Genus Calymnodon}

3.3.3.1. Calymnodon asiaticus Copel., Philipp. J. Sci. 38: 154. 1929. Type: Vietnam:
Annam: Mt. Bani, May-July 1927, J. \& M.S. Clemens 3800 (holotype UC 340509!; isotypes BM 001039898!, BM 001039897!, MO 022328!).- Laminae glabrous or sparcely hairy, narrowly elliptic, $2.5-4 \times 0.3-0.4 \mathrm{~cm}$, pinnately divided; pinnae 5-12 pairs; ca. 2-4 pairs of fertile pinnae at top of lamina, ovate, $1.5-2 \times 1.5 \mathrm{~mm}$, folded; ca. 3-8 pairs of sterile pinnae at base, oblong, ca. 2-3 x $0.9 \mathrm{~mm}$; hairs ca. 0.1-0.2 mm; sori protected by folded pinna, one per pinna; sori non sunken in pinna.- In heavily disturbed closed evergreen seasonal tropical broad-leaved submontane forest on foot of very steep mountain slopes composed with shale and granite along shady stream. Lithophytic on shady wet mossy stream boulders. Locally common.- CTFP. Thua Thien - Hue Prov., A Luoi Distr., Hong Van Comm., Ta Lo Vill. around point $16^{\circ} 21^{\prime} 52^{\prime \prime} \mathrm{N}, 107^{\circ} 09^{\prime} 36^{\prime \prime} \mathrm{E}$, at elevation about 1000-1100 m. Aver. et al. HAL 7661 B. 30 Apr 2005.

3.3.3.2. Calymnodon gracilis (Fée) Copel., Philipp. J. Sci., 34(3): 266-267. 1927. Basionym: Plectopteris gracilis Fée, Mém. Foug. 5: 230, pl. 19b. 1852 (Type not seen).Plants 3-4 cm tall, pendulous; laminae margin and both surfaces with frequent hairs, ca. $1 \mathrm{~mm}$ long, dark red-brown; fronds narrowly oblong or elliptic, 3-5 x 0.4-0.6 cm; pinnately divided; pinnae 7-22 pairs; ca. 5-7 pairs of fertile pinnae at top of lamina, narrowly obovate, ca. 3 x 1.3 $\mathrm{mm}$, folded; sterile pinnae at base, narrowly obovate, ca. 3 x $1.5 \mathrm{~mm}$; sori protected by folded pinna, one per pinna; sori non sunken in pinna, left from last year. Plants grow well.- STFP. Lam Dong Prov., Lac Duong Distr., Da Chays Comm., K'Long K'Lanh Vill., just behind the Hon Giao FP Station, $12^{\circ} 11^{\prime} 10^{\prime \prime} \mathrm{N}, 108^{\circ} 42^{\prime} 54^{\prime \prime}$ $\mathrm{E}$, at elevation $1640 \mathrm{~m}$; common, mixed with some filmy fern species on wet tree trunks and branchs in un- or slightly disturbed primary closed seasonal tropical montane cloud evergreen broad-leaved forest. Phan Ke Loc \& Quach Van Hoi P 11444. 03 Jan 2016.

\subsubsection{Genus Ctenopterella}

3.3.4.1. Ctenopterella nhatrangensis (C.Chr. \& Tardieu) Parris. Fern Gaz., 19(6): 207. 
2013. Basionym: Ctenopteris nhatrangensis C. Chr. \& Tardieu, Notul. Syst. Paris 8(4): 181. 1939. Type: Vietnam: Nhatrang, Song Ko. Poilane 3338 (BM 001039878!).- Fronds linearnarrowly lanceolate, ca. $5-9$ x $0.6-0.8 \mathrm{~cm}$, glabrous, pendulous, thin coriaceous, pinnate; stipes winged to base; obtuse to bluntly acute at apex, long-attenuate at base; pinnate to deeply divided to wing less ca. $0.5 \mathrm{~mm}$ wide along rachis; pinnae 16-35 pairs, longest ones in middle, oblong, ca. 2.5-4 x 1-1.5 mm, turning triangular to base, entire, bluntly obtuse at apex; sori 1.1-1.5 x 0.4-0.6 mm, broadly elliptic in outline, in two rows per pinna, one, rarely two on each side of the rachis, slightly sunken in broad shallow depressions which may appear slightly prominent on adaxial surface of lamina. In slightly disturbed primary closed seasonal tropical submontane evergreen broad-leaved forest; clustered in small groups on rocks along stream.- STFP. Khanh Hoa Prov., Khanh Vinh Distr., Son Thai Comm., around Cau Gop Dai and Tram Lam San (Forest Product Station) of Khanh Hoa Prov. Clustered in small groups on moss-covered trunk and branch bark, rarely on rocks, in slightly disturbed primary closed seasonal tropical submontane evergreen broadleaved forest. Plants grow very well. Other fern species rarely seen., nearby $12^{\circ} 11^{\prime} 10^{\prime \prime} \mathrm{N}$, $108^{\circ} 42^{\prime} 54$ " E, at elevation 1640 m. Phan Ke Loc \& Quach Van Hoi P 11430. 03 Jan 2016 \& Son Thai Comm., Gop Dai Bridge, $65 \mathrm{~km}$ E of Da Lat, $12^{\circ} 11^{\prime} 41^{\prime \prime} \mathrm{N}, 108^{\circ} 44^{\prime} 19^{\prime \prime} \mathrm{E}$, at elevation $1000-1100 \mathrm{~m}$. In primary heavily destroyed closed evergreen broad-leaved forests along granite stream banks. Rather common on destroyed rocks, rather dry. Other fern species rarely seen. Phan Ke Loc \& Quach Van Hoi P 11522. 01 May 2016.

\subsubsection{Genus Oreogrammitis}

3.3.5.1. Oreogrammitis congener (Blume) Parris. Gard. Bull. Singapore, 58: 257. 2007. Basionym: Grammitis congener Blume, Enum. Pl. Javae, 2: 115. 1828. Type: Blume s.n., Java (Type L!).- Fronds usually more than $8 \mathrm{~cm}$ long, $0.4-0.5 \mathrm{~cm}$ large; stipes $0.8-2.5 \mathrm{~cm}$, with dense red-brown hairs, ca. $1.5 \mathrm{~mm}$ long; laminae linear-elliptic, occasional oblanceolate, 7-12 x 0.4-0.6 cm, bluntly acute to acuminate or subrounded at apex, long-acuminate to attenuate at base, entire; with dark red-brown hairs 1.5-2 $\mathrm{mm}$ long, frequent on adaxial surface, especially amongst sori and on margin; midrib slightly prominent abaxially; sori \pm circular to broadly elliptic, 2.5-2.8 x 1.9-2.5 mm, oblique on surface of lamima, nearer to midvein than margin, discrete to confluent when mature; sporangia setose. It is difficult to identify those specimens with small fronds.- CTFP. Thua Thien-Hue Prov., Phu Loc Distr., Bach Ma National Park, S slope of Bach Ma mountain below Hai Vong Dai peak, to SW of point $16^{\circ} 11^{\prime} 41^{\prime \prime} \mathrm{N}, 107^{\circ} 51^{\prime} 26^{\prime \prime} \mathrm{E}$, at elevation 1100-1300 m, mixed and broadleaved evergreen secondary closed wet mountain forests on very steep shale/granite slopes. Lithophyte on wet mossy shady vertical rocks along stream. Not common. N.T.Hiep et al. HLF 1361.1 May 2002; Kon Tum Prov., Sa Thay Distr., Sa Nhon Comm., $14^{\circ} 27^{\prime}$ N, $107^{\circ} 45^{\prime}$ E, at elevation $1200 \mathrm{~m}$. In primary wet mossy mountain coniferous forest on sandstone. Very rare. PK Loc et al. P 7962. 23 Dec 1999; Kon Tum Prov., Kon Phong Distr., Hieu Comm., $14^{\circ} 39^{\prime} \mathrm{N}, 108^{\circ} 25^{\prime} \mathrm{E}, 1200 \mathrm{~m}$. in primary wet mossy dominants Pinus dalatensis, Fokienia hodginsii, Dacrydium elatum, Dacrycarpus imbricatus) along top ridges at elevation $1200 \mathrm{~m}$. Epiphyte on mossy trees in shady wet places. Very rare. Aver. et al. VH 5305. 18 Apr. 2000.SIFP. Dak Nong Prov., Dak Gloong Distr., Dak P'Lao Comm., steep mountain slopes along main ridge of Ta Dung mountain system at elevation 1700-1985 m, around point of highest peak at $11^{\circ} 51^{\prime} 30^{\prime \prime} \mathrm{N}, 108^{\circ} 02^{\prime} 08^{\prime \prime}$ E. Aver. et al. HLF 5620. 09 Nov 2005.

3.3.5.2. Oreogrammitis dorsipila (H.Christ) Parris, Gard. Bull. Singapore 58: 259. 2007. Basionym: Polypodium dorsipilum H.Christ, Monsunia 1: 59. 1900. Type: Unknown!; Tardieu \& C.Chr., Notul. Syst. (Paris) VIII: 179. 1939 \& Fl. Gén. Indo-Ch. 7, 2: 524. 1941.-Stipe $0.4-1 \mathrm{~cm}$, with dense simple solitary reddish brown hairs $0.2-0.5 \mathrm{~mm}$ long; lamina usually narrowly oblanceolate, $6-10 \times 0.4-0.8 \mathrm{~cm}$, 
decurrent to stipe, apex bluntly acute to obtuse; lateral veins hidden; hairs on all parts of lamina, simple, solitary, ca. 0.2-0.5 mm, dark reddish brown; sori orbicular or elliptic, superficial, close to midrib. In primary mossy wet coniferous forest with Fokienia hodginsii along main ridge. Epiphytic on mossy trees. Occasional. Note: Close to $O$. sinohirtella by lateral veins hidden, but differs by fronds bigger, up to $10 \times 0.8 \mathrm{~cm}$ and stipe hairs reddish brown and shorter, less than $0.5 \mathrm{~mm}$ long.- STFP. Lam Dong Prov., Lac Duong Distr., Da Chais Comm., around point $12^{\circ} 05^{\prime} 27^{\prime \prime} \mathrm{N} \mathrm{108} 39^{\prime} 43^{\prime \prime} \mathrm{E}$, at elevation 2100$2300 \mathrm{~m}$, along main ridge of Bi Doup mountain. Occasional. Aver. et al. HLF 5259. 15 Oct 2005 \& Aver. et al. HLF 5267a. 15 Oct 2005.

3.3.5.3. Oreogrammitis nuda (Tagawa) Parris., Gard. Bull. Singapore. 58: 264. 2007. Basionym: Grammitis nuda Tagawa, Acta Phytotax. Geobot. 10: 284. 1941. Type: Taiwan, Chinshui-yin-Tahanshan, Inter Sinsuiei et Daizyurin (Tahanlintao), 22 January 1939, Tagawa 2092 (holotype KYO!).- Fronds simple, glabrous, ca. 2-4 x 0.2-0.4 cm; laminae linear to linear-oblong, apex bluntly obtuse to subrounded, base attenuate or decurrent on stipe ca. $0.3 \mathrm{~cm}$ or sessile; midribs distinct and prominent on both sides; lateral veins hidden, simple, endings with hydathodes; sori medial or slightly nearer to midrib, \pm circular to broadly elliptic, oblique, sunken in broad shallow depressions on abaxial surface and distinctly prominent on adaxial surface of lamina. Epiphytic on wet tree trunks and branches in slightly disturbed primary closed seasonal tropical submontane cloud evergreen broadleaved forests. Rare. Note. The diagnostic character of this species is frond glabrous (vs. hairy in other species).- STFP. Lam Dong Prov., Lac Duong Distr., Da Chays Comm., K'Long K'Lanh Vill., behind of the Hon Giao Forest Protection Station, 12 ${ }^{\circ} 11^{\prime} 10^{\prime \prime}$ N, 108 $42^{\prime}$ '54" E, 1640 m, Phan Ke Loc \& Quach Van Hoi, P 11445. 03 Jan 2016; Khanh Hoa Prov., Khanh Vinh Distr., Son Thai Comm., Gop Dai Bridge, $57 \mathrm{~km}$ E of Da Lat, 12 $11^{\circ} 41^{\prime \prime} \mathrm{N}, 108^{\circ} 44^{\prime} 19^{\prime \prime} \mathrm{E}$, at elevation 1000-1100 m. Phan Ke Loc \& Quach Van Hoi P 11523. 01 May 2016.
3.3.5.4. Oreogrammitis reinwardtii (Blume) Parris. Gard. Bull. Singapore. 58: 266, 2007. Basionym. Grammitis reinwardtii Blume, Enum. Pl. Javae Add. 2. 1828; Polypodium reinwardtii (Blume) C. Presl., Tent. Pterid., 180. 1836. Type unknown.- Fronds covered by occasional to scattered dark reddish brown hairs, ca. 1.0-1.5 mm long; stipes ca. 0.2-0.6 cm, densely brown hairs, ca. $1 \mathrm{~mm}$ long; laminae narrowly elliptic, entire, sometimes undulate, $1.5-4 \times 0.3-0.6 \mathrm{~cm}$, gradually attenuate or decurrent at base; midrib and lateral veins slightly prominent on both surfaces; apex obtuse to rounded, midrib sometimes slightly sunken adaxially; lateral veins usually unforked, ending with hydathodes which may be evident only in young fronds; hairs occasional to sparse on both surfaces; sori orbicular or elliptic, superficial, close to midrib. Very rare, collected once with $O$. sinohirtella as lithophytic on stream banks under heavily disturbed primary closed evergreen broad-leaved submontane forest. In primary heavily destroyed closed evergreen broad-leaved forests along granite stream banks. Rare, on rocks, rather dry. Other fern species rarely seen there. Note: Differs from other species with laminae hairy by lateral veins distinct (vs. hidden in other species).- STFP. Khanh Hoa Prov., Khanh Vinh Distr., Son Thai Comm., Khanh Hoa Forest Productions Co, Protection \& F Manag. Station, at elevation 1500 $\mathrm{m}$, ca. $53 \mathrm{~km} \mathrm{E}$ of Da Lat, nearby $12^{\circ} 11^{\prime} 13^{\prime \prime} \mathrm{N}$, $108^{\circ} 42^{\prime} 55^{\prime \prime} \mathrm{E}$, at elevation $1634 \mathrm{~m}$. Other fern species rarely seen except Abrodictyum obscurum var. obscurum. Phan Ke Loc \& Quach Van Hoi P 11519. 01 May 2016.

3.3.5.5. Oreogrammitis sinohirtella Parris. Fl. China, 2-3: 842. 2013. Type: China. Guangdong: Ts'ung-hwa District, Cheung UK village, Sam Kok Shan, 1-16 Mar 1935, W. T. Tsang 24913 (holotype E 00194227!, isotype LU!).- Stipes covered by dense dark reddish brown hairs, ca. 1.0-1.5 mm long; laminae narrowly elliptic to oblanceolate, $2-5 \times$ x $0.15-0.5$ $\mathrm{cm}$, apex obtuse to acute, gradually attenuate at base; midrib slightly prominent abaxially; lateral veins invisible; sori orbicular or elliptic, 
superficial, close to midrib. The species of dwarf Grammitidoideae with the largest distribution (Ha Giang, Bac Thai, Quang Tri, Thua ThienHue, Dak Lak and Lam Dong). On moss-covered tree trunks and lithophytic on stream banks under disturbed primary closed evergreen seasonal tropical broad-leaved submontane forest. Note: The diagnostic character of this species is small sized and stipe hairs up to 1.8 mm long.- NEFP. Ha Giang Prov., Bac Me Distr., Phieng Luong Comm., around Phieng Luong Vill., about $4 \mathrm{~km}$ to SW $22^{\circ} 39^{\prime} 29^{\prime \prime} \mathrm{N}$, $105^{\circ} 19^{\prime} 03^{\prime \prime} \mathrm{E}$, at elevation 1150-1250 m. In slightly logged primary closed evergreen broadleaved forest on slopes of remnant mountains composed with solid highly eroded crystalline and partially shale limestone at elev. Epiphytic in shady place. Occasional. Aver. et al. HAL 6433. 4 Mar 2005; Bac Thai Prov., Dai Tu Distr., Ky Phu Comm., nearby 213' N, 105³9" E. VVChi s.n., 1967.- CTFP. Quang Tri Prov., Huong Hoa Distr., Huong Phung Comm., Sa Mu pass, around point $16^{\circ} 48^{\prime} 36^{\prime \prime} \mathrm{N}, 106^{\circ} 34^{\prime} 44^{\prime \prime} \mathrm{E}$, at elevation about 1300-1500 m, lithophytic and epiphytic in shady rocky edge of slightly disturbed closed evergreen shale mountain. N.T.Hiep et al. HLF 6283. 29 Mar 2006; Thua Thien-Hue, A Luoi, Hong Van Comm., Ta Lo Vill., around point $16^{\circ} 21^{\prime} 52^{\prime \prime} \mathrm{N}, 107^{\circ} 09^{\prime} 36^{\prime \prime} \mathrm{E}$ at elevation about 1000-1100 m. In secondary wet closed broad-leaved forest on very steep mountain slopes composed with shale and granite along shady stream canyon. Lithophyte on shady wet mossy stream boulders. Locally common. Aver. et al. HAL 7661. 30 Apr 2005.STFP. Lam Dong Prov., Lac Duong Distr., Da Chays Comm., just behind of the Hon Giao FP Station, 12 $11^{\circ} 10^{\prime \prime} \mathrm{N}, 108^{\circ} 42^{\prime} 54^{\prime \prime} \mathrm{E}$, at elevation 1640 m. Phan Ke Loc \& Quach Van Hoi P 11446. 03 Jan 2016.- SIFP. Dak Lak Prov., Krong Bong Distr., Cu Pui Comm., elevation from Dak Tour Vill., $12^{\circ} 30^{\prime} \mathrm{N}, 108^{\circ} 30^{\prime} \mathrm{E}$ to main peak. Aver. et al. VH 6124. 04 May 2000.

\subsubsection{Genus Scleroglossum}

3.3.6.1. Scleroglossum pusillum (Blume) Alderw. Bull. Jard. Bot. Buitenz. 2,7, 39, 1912. Basionym: Vittaria pusilla Blume, Enum. Pl.
Jav. 199, 1828. Type: Blume s.n., Java (holotype L not seen). Synonyms: Vittaria minor Fée, Mém. 3, 23, 1852; Scleroglossum minus (Fée) C.Chr., Dansk Bot.Arkiv 6, 29, 1929; Holttum, Rev.Fl.Malaya 2, 2nd ed., 236, 1968; Parris, Kew Bull.41, 515, 1936; Piggott, Fems Malay. 76, fig. 521-522, 1988; Tagawa \& Iwatsuki, Fl. Thailand 3, 599, fig. 59, 10, 1989. Type: Cuming 381 p.p., Peninsular Malaysia, Malacca (CGE).- Differs from species with soral grooves opening perpendicular to lamina (S. sulcatum) by soral grooves opening oblique to lamina. Differs from other species of the genus with soral grooves opening oblique to lamina ( $S$. puxidatum) is laminae not clearly narrowed below sori; fronds simple, glabrous; laminae 2.54 x 0.15-0.2 cm; sori linear, ca. 6-8 x $1.5 \mathrm{~mm}$. In primary slightly disturbed closed evergreen coniferous submontane forests along ridge tops on sandstone. Epiphytic on mossy trees in shady wet sites, occasional. Rare.- STFP. Lam Dong Prov., Lac Duong Distr., Da Chais Comm., around point $12^{\circ} 11^{\prime} 09^{\prime \prime} \mathrm{N} 108^{\circ} 41^{\prime} 20^{\prime \prime} \mathrm{E}$, at elevation about $1500 \mathrm{~m}$. In primary mixed forest with Pinus dalatensis and $P$. krempfii on $\mathrm{W}$ slope of Hon Giao ridge. T.T.T.Trang et al. HLF 5324. 20 Oct 2005.

3.3.6.2. Scleroglossum puxidatum Alderw. Bull. Jard. Bot. Buitenzorg, sér. 2 16: 37, t. 9. 1914. Type: INDONESIA. Borneo, Boekit Batoe-ayah, Nieuwenhuis 1647 (holotype BO!).Fronds simple, glabrous; laminae linear, subcoriaceous, $2.5-4 \times 0.2 \mathrm{~cm}$, clearly narrowed below sori. In primary slightly disturbed closed evergreen broad-leaved submontane forests along granite stream banks or in submontane and montane broad-leaved along ridge tops on sandstone. Epiphytic on mossy trees or lithophytic on large wet and shady mossy boulders and rocky cliffs. Rare. Differs from other species of the genus with soral grooves opening oblique to lamina. Rare.- STFP. Lam Dong Prov., Lac Duong Distr., Da Chais Comm., around point $12^{\circ} 05^{\prime} 27^{\prime \prime} \mathrm{N}, 108^{\circ} 39^{\prime} 43^{\prime \prime}$ E, at elevation 2100-2300 m, along main ridge of Bi Doup mountain in primary mossy wet coniferous forest with Fokienia hodginsii along 
main ridge. Lithophytic and epiphytic on mossy trees. Locally common. Aver. et al. HLF 5253. 15 Oct 2005.- SIFP. Dak Nong Prov., Dak Gloong Distr., Dak P'Lao Comm., steep mountain slopes along main ridge of Ta Dung mountain system at elevation 1700-1985 m, around point of highest peak at $11^{\circ} 51^{\prime} 30^{\prime \prime} \mathrm{N}$, $108^{\circ} 02^{\prime} 08^{\prime \prime}$ E, primary broad-leaved evergreen closed wet mossy cloudy forest on crystalline highly metamorphosed sandstone. Epiphyte on mossy trees and lithophyte on large mossy boulders and rocky cliffs. Common. Aver. et al. HLF 5655. 8 Nov 2005.

3.3.6.3. Scleroglossum sulcatum (Kuhn) Alderw. Bull. Jard. Bot. Buitenzorg, sér. 2 7: 37, pl. 5, f. 3-4. 1912. Basionym: Vittaria sulcata Kuhn, Linnaea 36: 68. 1869. Type: SRI LANKA, Thwaites 3807 (paralectotype B!; isolectotype B!, isosyntype GH!). Synonym: Taeniopsis sulcata (Kuhn) Bedd., Suppl. Ferns Brit. Ind. 25. 1876.- Fronds simple, glabrous; laminae linear, subcoriaceous, $2.5-4$ x $0.2 \mathrm{~cm}$; sori linear, ca. $4-14 \times 1.5 \mathrm{~mm}, \pm$ parallel to midvein and sunken in lamina. In primary heavily destroyed closed evergreen broad-leaved submontane forests along granite stream banks or in submontane coniferous along ridge tops on sandstone. Rare, on rocks rather dry or epiphyte on mossy trees in shady wet sites. Other fern species rarely grown. Occasionally common. The diagnostic character of this species is soral grooves opening perpendicular to lamina (vs. soral grooves opening oblique to lamina in other species).- CTFP. Kon Tum Prov., Kon Plong Distr., Hieu Comm., Mang La forest enterprise, $14^{\circ} 39^{\prime} \mathrm{N}, 108^{\circ} 25^{\prime} \mathrm{E}$, at elevation $1100-1200 \mathrm{~m}$. Aver. et al. VH 5531; Kon Tum Prov., Kon Plong Distr., Hieu Comm., Mang La forest enterprise, $14^{\circ} 39^{\prime} \mathrm{N}, \quad 108^{\circ} 25^{\prime} \mathrm{E}$, at elevation $1200 \mathrm{~m}$. Epiphytic on mossy trees inshade wet places. In primary wet mossy mountain coniferous forest on sandstone (with Pinus dalatensis, Fokienia hodginsii, Dacrydium elatum, Dacrycarpus imbricatus) along top ridge. Aver. et al. VH 5304. 18 Apr 2000.- Kon Tum Prov., Kon Plong Distr., Hieu Comm., Mang La forest enterprise, $14^{\circ} 39^{\prime} \mathrm{N}, 108^{\circ} 25^{\prime} \mathrm{E}$, at elevation $1100-1200 \mathrm{~m}$. In primary broad-leaved and mixed (with Dacrydium and Dacrycarpus) evergreen dry mountain forest at elevation about 1100-1200 m on sandstone. Epiphytic on high mossy tree in shady place. Not common. Aver. et al. VH 5531. 21 Apr 2000.- STFP. Khanh Hoa Prov., Khanh Vinh Distr., Son Thai Comm., Khanh Hoa Forest Productions Co, Protection \& F Manag. Station, at elevation $1500 \mathrm{~m}$, ca $53 \mathrm{~km} \mathrm{E}$ of Da Lat, $12^{\circ} 11^{\prime} 13^{\prime \prime} \mathrm{N}, 108^{\circ} 42^{\prime} 55^{\prime \prime} \mathrm{E}$, at elevation $1634 \mathrm{~m}$. In primary heavily destroyed closed evergreen broad-leaved forests along granite stream banks. Rare, on rocks, rather dry. Other fern species rarely seen except Abrodictyum obscurum var. obscurum. Phan Ke Loc \& Quach Van Hoi P 11520. 01 May 2016 and Aver. et al. VH 5304. 21 Apr 2000.

\subsubsection{Genus Tomophyllum}

3.3.7.1. Tomophyllum repandulum (Mett.) Parris. Gard. Bull. Singapore, 58(2): 248. 2007. Basionym: Polypodium repandulum Mett., Abh. Senckenberg. Naturf. Ges. 2(1): 50. 1856. Type: Sril Lanka, Gardner 58 (BM, OXF not seen).Rhizome radial, stipes in whorls; fronds thin coriaceous, glabrous on abaxial surface, hairs occasional to sparse on adaxial surface of midvein, narrowly elliptic in outline, 6-8 cm long, $0.7-0.8 \mathrm{~cm}$ wide in middle, narrower to top and base, pinnately lobed; wing ca. 1-1.5 mm along rachis; pinnae 14-20 pairs, medium oblong, turning triangular to base; sori not protected, superficial or slightly sunken on abaxial surface and slightly prominent on adaxial face, in two rows per pinna, one each side per pinna midvein, or one sorus per pinna. In slightly disturbed primary evergreen closed seasonal tropical lowland broad-leaved forest on rocky mountain slopes composed with granite. Creeping lithophytic fern on wet and shady rocks, along stream. Not common.- SIFP. Ninh Thuan Prov., Ninh Hai Distr., Vinh Hai Comm., NEE slopes on Nui Chua mountain to SW of $11^{\circ} 43^{\prime} 52^{\prime \prime} \mathrm{N}, 109^{\circ} 08^{\prime} 35^{\prime \prime} \mathrm{E}$, at elevation of 800$900 \mathrm{~m}$, in primary evergreen closed dry forests on rocky mountain slopes composed with granite. J.Regalado et al. HLF 4381. 28 Mar 2004 \& J.Regalado et al. HLF 4448. 31 Mar 2004. 


\section{Conclusion}

Goal of our study is making a detailed checklist of 43 grammitoid collecting numbers kept in HNU, the Herbarium of the Biological Museum of the VNU University of Science (HUS). Traditional morphological methods are used. PPG I (2016) is adopted in classifying specimens. 15 references are used to identify their scientific names. Carefully field labels enable rich and diverse data to make detailed checklist. Their geographical distribution is arranging in Floristic Provinces.

As the results 43 collected numbers belong to 16 species in 7 genera. Data of each species are presented in following order: Nomenclature section, morphological description, habitat and ecology, geographical distribution in Floristic Province.

The richest in number of species is Oreogrammitis with 5 species; two genera (Prosaptia and Scleroglossum)- each with 3 species, one genus (Calymnodon) with two species; other genera (Cteropterella, Themelium and Tomophyllum)- each with one species.

\section{Acknowledgements}

Thanks are due to HNU?, the herbarium of the Biological Museum, VNU University of Science (HUS) for enabling us to study the specimens there, to Parris B.S. for identifying $P$ 11430 and $P \quad 11522$ as Cteroptenella nhatrangensis Parris.

\section{References}

[1] PPG I, The Pteridophyte Phylogeny Group, A community-derived classification for extant lycophytes and ferns. Fam. PolypodiaceaeSubfam. Grammitidoideae. J. Syst. Evol. 54(6) (2016) 592-594.

[2] B.S. Parris, R. Kiew, R.C.K. Chung, L.G. Saw, E. Soepadmo, Flora of Peninsular Malaysia. Series I: Ferns and Lycophytes, Vol. 1. Malayan Forest
Records No 48 Ministry of Science, Technology and Innovation, Government of Malaysia, 2010.

[3] L.V. Averyanov, P.K. Loc, N.T. Hiep, D.K. Harder, Phytogeographic review of Vietnam and adjacent areas of Eastern Indochina, Komarowia 3 (2003) 20-21.

[4] C.E. DeVol, C.M. Kuo, W.C. Shieh, J.L. Tsai, T.Y. Yang, In Flora of Taiwan, second ed. Vol. 1. Pteridophyta, Gymnospermae, Editorial Committee of the Flora of Taiwan Taipei, Taiwan, 1994, pp. 23-559.

[5] B.S. Parris, W.C. Cheng, C.H. Tian, L.T. Ngan, NQ. Dat, L.H. Truong, New species and records of grammitid ferns (Polypodiaceae) for Vietnam, Phytotaxa 266 (1) (2015) 39.

[6] P.H. Ho, An Illustrated Flora of Vietnam, Vol. I, part 1: 18-261. Montréal-published by the author, 1991.

[7] P.K. Loc, The Updated Checklist of the Fern Flora of Vietnam following the classification scheme of A. Smith et al. J. Fairylake Botanical Garden 9 (3) (2010) 1-13.

[8] M. Tagawa, K. Iwatsuki, Flora of Thailand, Vol. 3, parts 1. Pteridophytes, The Forest Herbarium, National Park, Wildlife and Plant Conservation Department, Bangkok, 1979.

[9] M. Tagawa, K. Iwatsuki, Flora of Thailand, Vol. 3, parts 2. Pteridophytes, The Forest Herbarium, National Park, Wildlife and Plant Conservation Department, Bangkok, 1985.

[10] M. Tagawa, K. Iwatsuki, Flora of Thailand, Vol. 3, parts 3. Pteridophytes, The Forest Herbarium, National Park, Wildlife and Plant Conservation Department, Bangkok, 1988.

[11] M. Tagawa, K. Iwatsuki, Flora of Thailand, Vol. 3, parts 4., Pteridophytes. The Forest Herbarium, National Park, Wildlife and Plant Conservation Department. Bangkok, 1989.

[12] M.L. Tardieu-Blot, C. Christensen, A. Alston, Flore Générale de l'Indo-Chine, Tome 7, partie 2, Cryptogames vasculaires, Masson et Cie Éditeurs, Paris. 1939-1951

[13] Z.Y. Wu, P.H. Raven, D.Y. Hong, (eds.), Flora of China- Lycopodiaceae through Polypodiaceae, 23. Science Press (Beijing) \& Missouri Botanical Garden Press (St. Louis), 2013a.

[14] Z.Y. Wu, P.H. Raven, D.Y. Hong, (eds.), Flora of China Illustrations- Lycopodiaceae through Polypodiaceae. 2-3. Science Press (Beijing) \& Missouri Botanical Garden Press (St. Louis), 2013.

[15] B.S. Parris, Themelium a new genus of Grammitidaceae (Filicales), Kew Bull., 52 (3) (1997) 737-738. 